\title{
WLAN-Reichweite clever erhöhen
}

\author{
Ein Praxis-WLAN ist doppelt interessant: Es kann ein guter Zusatz- \\ service für die Patienten sein. Es bietet aber auch Ärzten und MFA \\ mehr Freiraum - etwa bei der Dokumentation. Doch was, wenn die \\ Reichweite des kabellosen Web-Anschlusses nicht mitspielt?
}

$\mathrm{D}$ ie Basisstation von Praxis- oder Klinik-WLAN befindet sich in der Regel dort, wo auch der Telefon- oder DSL-Anschluss installiert ist häufig also an der Rezeption oder auf dem Gang. Je nach Größe, Grundriss und baulicher Beschaffenheit der zu versorgenden Räumlichkeiten kann es bei größerem Abstand zur Basis mit dem WLAN-Empfang eng werden. Auch wenn die WLAN-Reichweite gern mit 300 Metern angegeben wird, ist dies häufig ein rein theoretischer Wert. Wände, Möbel und sogar Pflanzen schirmen die Funksignale mehr oder weniger stark ab, sodass das Funknetz tatsächlich schon nach 50 Metern oder weniger verstummen kann.

\section{Verbindung per Knopfdruck}

Die einschlägigen Hersteller haben dieses Problem erkannt und bieten dafür eine Lösung an: WLAN-Repeater, bisweilen auch "Range Extender" genannt, verstärken das Funksignal. Zur Installation genügt es meist, den Repeater in eine Steckdose zu stecken und mit der Basisstation zu koppeln. Dies geht besonders komfortabel, wenn WLAN-Basis und Repeater den WPS-Modus unterstützen. "WiFi Protected Setup“ (WPS) steht für die Möglichkeit, eine verschlüsselte Verbindung zwischen Geräten einfach per Knopfdruck herzustellen. Nach Aktivierung dieser Funktion an der Basis schaltet diese für eine $\mathrm{Mi}$ nute in einen Anmeldemodus um. Wird innerhalb dieser Zeit auch die WPS-Taste am Repeater gedrückt, akzeptiert die Basis dieses Gerät und meldet es dauerhaft an. Die Verbindung zwischen beiden Geräten erfolgt dabei mit der star- ken Verschlüsselung „WPA2“ - eine unverschlüsselte Verbindung wäre fahrlässig, da dann auch unberechtigte Geräte in das WLAN eindringen könnten.

\section{Ein Repeater für alle Netze}

In der Theorie sollten Repeater, Router oder WLAN-Basisstationen auch von unterschiedlichen Herstellern zusammenarbeiten können. In der Praxis empfiehlt es sich, beide Geräte vom gleichen Anbieter zu beziehen, da dies das Risiko von Unverträglichkeiten oder nicht vollständig unterstützten Funktionen verringert. Wird zum Beispiel neben dem abgeschlossenen Praxis- oder KlinikNetz auch ein offenes Patienten-WLAN betrieben, sollte der Repeater beide Netze weiterleiten. Dies ist keineswegs bei allen Modellen gewährleistet.

Der Repeater muss an einem Ort aufgestellt werden, an dem das Signal der WLAN-Basis noch gut empfangen wird. Hat der Repeater die Signale der Basis aufgefangen, „wiederholt" er sie, sodass das aufgefrischte Signal ab diesem Standort wieder mit vollem Pegel ausgestrahlt wird. Je nach Aufstellungsort lässt sich die Reichweite somit in der Richtung der Achse zwischen Basis und Repeater annähernd verdoppeln. Die beschriebene Funktionsweise hat jedoch ihre Tücken: Da das Ursprungssignal der Basis und das vom Repeater neu ausgestrahlte Signal beide im gleichen Frequenzbereich liegen, halbiert sich de facto die Übertragungskapazität - also die WLAN-Geschwindigkeit. Wenn das Signal ohnehin schwach ist und/oder viele Nutzer im Funknetz angemeldet sind, kann diese Einschränkung empfindlich sein.

\section{Geschwindigkeit im Blick}

Nun haben aber Hersteller wie AVM, Asus, TP-Link oder Netgear die Repeater noch intelligenter gemacht: „Dualband-Repeater" unterstützen - wie es auch bei WLAN-Routern verbreitet ist zwei verschiedene Frequenzbänder: 2,4 und $5 \mathrm{GHz}$. Während das 2,4-GHzBand schon seit Anbeginn der WLANTechnik in den 1990er-Jahren genutzt wird, kam $5 \mathrm{GHz}$ um die Jahrtausendwende dazu. Nach wie vor ist daher das 2,4-GHz-Band gerade in dicht besiedelten Gebieten stärker ausgelastet als das 5-GHz-Band. Doch seit etwa fünf bis acht Jahren können viele modernere Endgeräte wie Smartphones, Tablets oder Notebooks auch das 5-GHz-Band nutzen.

Während die Dualband-Funktion noch recht verbreitet ist, gehen manche Anbieter wie die Berliner Firma AVM noch einen Schritt weiter: Ihre Repeater nutzen eine Strategie, die als „Crossband Repeating" bezeichnet wird: Bucht sich ein Endgerät zum Beispiel im 2,4-GHzNetz des Repeaters ein, wird für die Verbindung zwischen Basis und Repeater das 5-GHz-Band genutzt - und umgekehrt. Da der Empfang der WLAN-Signale und deren Weiterverbreitung somit nicht auf demselben Funkkanal erfolgen muss, lässt sich die bei einem „Sameband Repeating“ sonst zwangsläufig stattfindende Halbierung der Übertragungsgeschwindigkeit vermeiden. Der Repeater entscheidet für jeden angemeldeten WLAN-Client, welcher Frequenzbereich für die WLAN-Versorgung sowie für die WLAN-Strecke zum Router am besten geeignet ist. Zudem unterscheidet auch der Repeater zwischen Praxis-Netz und Patienten-Gastnetz, sofern beide Netze eingerichtet sind. Auch wenn viele Clients angemeldet sind, steigern diese Funktionen sowohl Reichweite als auch Übertragungsgeschwindigkeit.

Hannes Rügheimer 